\title{
Características de los ingresantes a la carrera de Medicina Veterinaria y Zootecnia de la Universidad Peruana Cayetano Heredia en el periodo 2010-2013
}

Ana Paredes ${ }^{1}$, José Zuazo ${ }^{1}$

\begin{abstract}
RESUMEN
El interés por la educación superior en el país ha ido incrementando con los años y junto con ella la competencia entre los centros que la ofrecen. La carrera de Medicina Veterinaria y Zootecnia tiene la misma tendencia creciente por lo que se hace necesario focalizar los esfuerzos de marketing en el potencial grupo poblacional que está dispuesto a estudiar la carrera y permita centrar los esfuerzos publicitarios ahorrando tiempo y dinero. En ese contexto el objetivo del estudio fue determinar las características de los ingresantes a la Facultad de Medicina Veterinaria y Zootecnia de la Universidad Peruana Cayetano Heredia (FAVEZ - UPCH) durante el periodo 2010- 2013 utilizando la base de datos de la Oficina de Admisión Matricula y Registro Académico (OFAD) de la universidad. Para ello se elaboró una base de datos utilizando el programa Microsoft Excel. La información fue resumida en base a estadística descriptiva y la asociación entre las variables fue analizada mediante la prueba de Chi Cuadrado. Se encontró que la población ingresante tuvo una tendencia creciente, y que el género predominante significativamente fue el femenino (74\%), el 92,4\% nacieron en Lima, y 98,2\% tienen nacionalidad peruana, el 30,3\% vive en Lima Centro, la edad de ingreso en promedio fue de 18,12 años, el $13,4 \%$ provinieron de un colegio nacional y el $86,6 \%$ de un colegio particular, la modalidad de ingreso de mayor preferencia fue la de factor excelencia con un 56,3\%, y el 29,2\% no tienen ningún tipo de beca. Los resultados encontrados representan una base para la caracterización de los potenciales postulantes a la carrera de Medicina Veterinaria y Zootecnia en la FAVEZ-UPCH.
\end{abstract}

Palabras claves: FAVEZ, UPCH, ingresantes, Medicina Veterinaria.

\section{INTRODUCCION}

La educación superior en el Perú es una opción de educación adicional para quienes han completado la educación básica. El interés por ella en la población mayor de 15 años ha crecido de $1 \%$ en 1940 a casi $25 \%$ en 2005. Así, mientras en 1970 los postulantes universitarios representaron $4,4 \%$ de la población de 17 a 20 años, en el año 2005 representaron 12,7\% (Díaz, 2008). La preferencia por los estudios universitarios también se encuentra asociado al nivel socioeconómico (NSE). Para el año 2006 los jóvenes limeños (15-18 años) de NSE A, B y C tuvieron algún tipo de interés en postular a una universidad, mientras que el resto pensó postular a un instituto, principalmente los de NSE C (APOYO S.A., 2006).

En 1980 existían 35 universidades (25 públicas y 10 privadas); mientras que para el año 1994 había 56 universidades (28 públicas y 28 privadas), duplicándose el número de universidades y de alumnos (Ministerio de Educación, 2006). Para el 2013, el país contó con 128 universidades: en Lima 44 privadas y 10 públicas, en el Norte 16 privadas y 9 públicas, en el Sur 14 privadas y 14 públicas y en el Centro y Oriente 10 privadas y 11 públicas (Grupo Educación al Futuro

\footnotetext{
Facultad de Medicina Veterinaria y Zootecnia, Universidad Peruana Cayetano Heredia
} 
e Investy S.A.C., 2013). Este crecimiento se produjo tras la aparición del Decreto Legislativo 882 donde se permitía que las universidades privadas funcionen como empresas con fines de lucro (Díaz, 2008).

La preferencias de las carreras por parte de los jóvenes limeños en el 2011 recayó sobre la carrera de Educación, Ingeniería, Ciencias Contables y Financieras, Administración de Empresas, Derecho y Ciencias Políticas, y en menor porcentaje la carrera de Enfermería, Biología, Medicina Humana, Economía, Medicina Veterinaria y Zootecnia (MVZ), Psicología, y Ciencias de la Comunicación; aunque esta preferencia se viene incrementando (INEI, 2013).

Centrados en la Carrera de MVZ, un estudio realizado en México sobre 566 estudiantes concluyó que el $83,3 \%$ de los participantes escogieron la carrera de MVZ como primera opción de estudios superior (Ortega, 2011). La elección se explicaba como una fuerza directa del área en que los estudiantes se comprometen y esfuerzan para lograr altos niveles de desempeño (Mikkonnen et al., 2009) y su interés ya sea por el contenido curricular mismo o por la atracción hacia un campo de trabajo determinado (Ortega, 2011).

El Médico Veterinario o Médico Veterinario Zootecnista debe estar preparado para desempeñarse en medicina veterinaria internacional y conocer de las interrelaciones subregionales y regionales de la salud animal, el medio ambiente, biodiversidad y la salud pública en general, con el propósito de promover la cooperación en la prevención, control, y la erradicación de las enfermedades transfronterizas de los animales (Taylor, 2012).

Esta perspectiva de la carrera a futuro ha llevado a un incremento de centros de estudios superior que ofrecen las profesiones Médico Veterinarias, con el consecuente aumento de la competencia. Debido a ello, la Facultad de Medicina Veterinaria y Zootecnia de la Universidad Peruana Cayetano Heredia (FAVEZUPCH) compite con otras instituciones privadas en la captación y fidelización de estudiantes. Dado que la permanencia de los estudiantes a lo largo de la carrera es importante, especialmente en las universidades de carácter privado, es que se debe realizar un diagnóstico inicial que abarque la realidad socioeconómica y cultural como contexto de procedencia del ingresante a la universidad (Bertoni, 2005). Ello mismo servirá de insumo para focalizar los esfuerzos de marketing en el potencial grupo poblacional que está dispuesto a estudiar la carrera y permita centrar los esfuerzos publicitarios ahorrando tiempo y dinero. Por ello, el objetivo del estudio fue determinar las características de los ingresantes a la FAVEZ-UPCH durante el periodo 2010- 2013.

\section{MATERIAL Y MÉTODOS}

Se estudió la población de ingresantes a la carrera de Medicina Veterinaria de la FAVEZ - UPCH en el periodo 2010 a 2013, mediante un análisis descriptivo retrospectivo. El estudio se realizó en base a la información brindada por la Oficina de Admisión, Matrícula y Registro Académico (OFAD), y la Facultad de Veterinaria y Zootecnia (FAVEZ) UPCH.

Las variables utilizadas en el estudio fueron:

1) Año de ingreso.

2) Modalidad (factor excelencia; exonerado, titulados o graduados; por proceso de admisión; traslado externo; traslado interno, admisión extraordinaria por deportista calificado, centro pre universitario).

3) Datos demográficos del ingresante (edad, sexo, lugar de nacimiento, lugar de residencia).

4) Tipo de colegio de procedencia (particular y nacional).

5) Beca de subvención económica asignada.

6) Datos de los padres (Ocupación).

Los datos extraídos fueron trasladados a una base de datos en el programa Microsoft Excel. La variable cuantitativa continua edad de los estudiantes fue resumida mediante estadística descriptiva utilizando la media como medida de tendencia central, y desviación estándar y rango como medida de dispersión. Las variables de clasificación fueron resumidas en cuadros expresando los resultados en frecuencias absolutas y relativas. La diferencia entre las edades, según variable de clasificación contrastada se evaluó mediante la prueba de T de Student, y la asociación entre las variables cualitativas fue evaluada mediante la prueba de Chi cuadrado.

Para el desarrollo del estudio se solicitó la aprobación del Comité Institucional de Ética (CIE) de la UPCH. Asimismo, se solicitó las autorizaciones correspondientes al Responsable de la OFAD y a las autoridades académicas de la FAVEZ.

\section{RESULTADOS}

El estudio recolectó información de 277 ingresantes a la FAVEZ - UPCH durante el periodo 2010-2013. Se 
observó una tendencia creciente de ingresantes en los años de estudio. Aunque la población masculina tuvo una conducta creciente con respecto al primer año del estudio, las mujeres predominaron significativamente durante el periodo evaluado $(\mathrm{p}<0,05)$ (Cuadro 1$)$.

El 98,2\% de ingresantes fueron de nacionalidad peruana. La mayoría de ingresantes tuvieron como lugar de residencia el departamento de Lima $(92,4 \%)$. Los demás procedían del interior del país $(6,2 \%)$ y el extranjero (1,4\%). El lugar de residencia de la familia de los ingresantes fueron mayoritariamente los distritos del cono centro, norte, sur y este con 30,3\%, $29,2 \%, 14,8 \%$ y $10,8 \%$ respectivamente. La diferencia corresponde a otros departamentos del interior del país $(7,6 \%)$, Callao $(5,1 \%)$ y provincias de Lima $(2,2 \%)$.

La mayoría de ingresantes provinieron de colegios particulares y la modalidad de ingreso más utilizada fue la de factor excelencia, seguida por la de examen de admisión, sin embargo se observó un importante porcentaje de ingresantes por modalidad de traslado

Cuadro 1. Distribución según género respecto al año de ingreso de la Facultad de Medicina Veterinaria y Zootecnia de la Universidad Peruana Cayetano Heredia. Periodo 2010- 2013 (n=277).

\begin{tabular}{ccccccc}
\hline \multirow{2}{*}{ Año de ingreso } & \multicolumn{2}{c}{ Total } & \multicolumn{2}{c}{ Sexo } & \multicolumn{2}{c}{ Sexo } \\
& ingresantes & \multicolumn{2}{c}{ femenino } & \multicolumn{2}{c}{ masculino } \\
& Nro. & $\%$ & Nro. & $\%$ & Nro. & $\%$ \\
\hline 2010 & 50 & 18,1 & 37 & 74,0 & 13 & 26,0 \\
2011 & 55 & 19,8 & 42 & 76,4 & 13 & 23,6 \\
2012 & 77 & 27,8 & 54 & 70,1 & 23 & 29,9 \\
2013 & 95 & 34,3 & 72 & 75,8 & 23 & 24,2 \\
Total & 277 & 100,0 & 205 & 74,0 & 72 & 26,0 \\
\hline
\end{tabular}

Cuadro 2. Distribución de los ingresantes a la Facultad de Medicina Veterinaria y Zootecnia de la Universidad Peruana Cayetano Heredia según colegio de procedencia, modalidad de ingreso y beca asignada. Periodo $2010-2013(\mathrm{n}=277)$.

\begin{tabular}{cccc}
\hline Variable & Estrato de la variables & Número & Porcentaje \\
\hline Tipo de & Particular & 240 & 86,6 \\
colegio & Nacional & 37 & 13,4 \\
& Factor Excelencia & 156 & 56,3 \\
& Admisión General & 70 & 25,3 \\
Modalidad de & Traslado Externo & 31 & 11,2 \\
ingreso & Traslado Interno & 5 & 1,8 \\
& Exonerado Titulados y Graduados & 2 & 0,7 \\
& Admisión extraordinaria & 1 & 0,4 \\
& Centro Pre Universitario & 12 & 4,3 \\
& Sin becas (0\%) & 81 & 29,2 \\
Tipo de & Entre 1 - 10\% & 14 & 5,1 \\
Beca & Entre 11 - 20\% & 24 & 8,7 \\
& Entre 21 - 30\% & 41 & 14,8 \\
& Entre 31 - 40\% & 36 & 13,0 \\
& Entre 41 - 50\% & 59 & 21,3 \\
& Entre 51 - 60\% & 14 & 5,1 \\
& $80 \%$ a más & 5 & 1,8 \\
& Sin Información & 3 & 1,1 \\
\hline
\end{tabular}


externo, que relega a la modalidad de ingreso por el centro pre universitario. La distribución de las becas tuvo un comportamiento más irregular, siendo mayor la población de los ingresantes sin becas $(29,2 \%)$ (Cuadro 2).

La relación entre la modalidad de ingreso y el tipo de colegio de procedencia muestra que para la población proveniente de colegio nacional la modalidad por factor excelencia y admisión general son utilizadas casi en el mismo porcentaje $(40,5 \%$ y $37,8 \%$ respectivamente) y para la población de colegio particular la diferencia es casi el doble $(58,8 \%-23,3 \%$ respectivamente) (Cuadro 3 ).

La edad de ingreso a la universidad fue en promedio 18,12 años, con una desviación estándar de 2,54 años (valor mínimo de 15 y valor máximo de 30 años). La población proveniente de estudios particulares presenta una edad promedio de ingreso más baja que aquella proveniente de estudios nacionales, al igual que el sexo femenino respecto al masculino (Cuadro 4).

La relación entre el sexo y colegio de procedencia revelaron que, si bien la mayor parte de la muestra total era de sexo femenino, al ser comparado con el tipo de colegio de procedencia, la población proveniente de colegio nacional presenta una diferencia poco característica entre el sexo femenino y masculino $(59,5 \%$ - 40,5\% respectivamente), a diferencia de la población proveniente de colegio particular que muestra una diferencia más resaltante siendo el 76,3\% sexo femenino.

Cuadro 3. Número y porcentaje de ingresantes según modalidad de ingreso y tipo de colegio de procedencia de los ingresantes de la Facultad de Medicina Veterinaria y Zootecnia de la Universidad Peruana Cayetano Heredia. Periodo $2010-2013(\mathrm{n}=277)$.

\begin{tabular}{ccccc}
\hline \multirow{2}{*}{ Variables } & \multicolumn{3}{c}{ Tipo de Colegio } \\
Modalidad de Ingreso & \multicolumn{2}{c}{ Nacional } & Particular \\
\cline { 2 - 5 } Factor Excelencia & Nro. & $\%$ & 141 & $\%$ \\
\hline Exonerado Titulado y Graduado & 15 & 40,5 & 1 & 58,8 \\
Admisión General & 1 & 2,7 & 56 & 0,4 \\
Traslado Externo & 14 & 37,8 & 23,3 \\
Traslado Interno & 6 & 16,2 & 4 & 10,4 \\
Admisión Extemporánea & 1 & 2,7 & 1 & 1,7 \\
Centro Pre Universitario & -- & -- & 12 & 0,4 \\
TOTAL & -- & -- & 240 & 5 \\
\hline
\end{tabular}

Cuadro 4. Edad promedio según sexo y tipo de colegio de procedencia de los ingresantes de la Facultad de Medicina Veterinaria y Zootecnia de la Universidad Peruana Cayetano Heredia. Periodo 2010 - 2013 $(\mathrm{n}=277)$.

\begin{tabular}{cccc}
\hline Tipo de Colegio & \multicolumn{2}{c}{ Edad \pm DE } & Total \\
\cline { 2 - 3 } & Mujer & Hombre & \\
\hline Nacional & $18,5 \pm 2,9$ & $19,3 \pm 3,8$ & $18,8 \pm 3,2$ \\
Particular & $18,0 \pm 2,4$ & $18,1 \pm 2,4$ & $18,0 \pm 2,4$ \\
Total & $18,0 \pm 2,5$ & $18,4 \pm 2,8$ & $18,1 \pm 2,5$ \\
\hline
\end{tabular}




\section{DISCUSIÓN}

El estudio encontró que el número de ingresantes anual a la FAVEZ UPCH durante el periodo de estudio, ha ido incrementado, tal es así que si se compara el número de ingresantes del año 2010 con los ingresantes del año 2013, este número casi se ha duplicado. A nivel nacional, según el último censo universitario realizado por el INEI, la población de pre grado de las universidades privadas tiene una tasa de crecimiento de un 9,3\% anual, a diferencia de las públicas que sólo llega al 3,2\% anual. A pesar del crecimiento de la competencia universitaria, la UPCH tuvo un incremento de $4,0 \%$ anual de la población universitaria de pre grado en el periodo 1996-2010 (INEI, 2011). Debido a estas evidencias de crecimiento, para la FAVEZ-UPCH se estima que en el siguiente quinquenio exista un aumento del $20 \%$ de ingresantes a la carrera, según el último plan operativo desarrollado para la facultad.

Las características demográficas tuvieron una distribución poco homogénea. El género femenino $(74,0 \%)$ sobrepasó en gran porcentaje al género masculino $(26,0 \%)$ y es que la presencia de la mujer en la educación universitaria del Perú se ha incrementado de manera continua desde la década de 1960, para el caso de ingresantes a nivel nacional, las mujeres representaron el $27 \%$ del total, y para el año 2004 fueron el 45,8\% (Díaz, 2008). La misma tendencia se manifestó en este estudio, ya que para el año 2010 tuvo una población de 37 mujeres ingresantes y 72 para el 2013. Esta tendencia no solo se evidenciada en nuestro país, la carrera de medicina veterinaria ha tenido un aumento indudable de la población femenina; a pesar de ello no existe un factor claro que explique este cambio, pero se le podría atribuir a un mayor sensibilidad que presenta el sexo femenino probablemente relacionado a una tendencia de cuidado y protección hacia el más débil, según la neurociencia, además de su capacidad por desarrollar tareas motoras delicadas y su densidad de atención difusa aunque más ancha (abarca más elementos; Pallarés, 2011).

Una de las características demográficas que mantiene la misma tendencia a lo largo de los años es la edad de los ingresantes. La edad promedio que se obtuvo fue de 18,12 años (desviación estándar de 2,54). En condiciones ideales, el ingreso a la universidad debería darse al año siguiente de egresar de la secundaria, siendo la edad normativa de culminación de la secundaria los 16 años, el ingreso a la universidad sería hacia los 17 años de edad. Sin embargo en la práctica no siempre se espera lo mismo, para este estudio el $48 \%$ tenían 18 años a más, esto podría deberse a diferentes situaciones como el tiempo invertido en la preparación para ingresar a la universidad, la falta de éxito de lograr el ingreso, la necesidad de buscar un trabajo para costear sus estudios, haber tenido estudios previos, entre otras (INEI, 2011).

La UPCH para el año 2010 tuvo 33,8\% ingresantes menores de 20 años de edad, $49,2 \%$ de 20 a 24 años y $17,1 \%$ mayores de 25 años; siendo la edad promedio 18,2 , muy similar a la edad promedio del ingresante universitario a nivel nacional (18,9 años). Esto demuestra que la edad es un dato que mantiene la misma tendencia a nivel nacional, sin importar la carrera universitaria. Como dato adicional se evaluó la edad promedio de ingreso a la FAVEZ del sexo femenino y masculino respecto al tipo de colegio de procedencia, los resultados obtenidos fueron similares a la tendencia nacional publicados por el INEI en el 2011, para ambos casos la edad promedio fue mayor en hombres que en mujeres y la población proveniente de colegio particular tuvo una media menor a la de colegio nacional; sin embargo estos datos no son representativos ya que la diferencia máxima obtenida fue sólo de 0,8 años.

Adicional a estas variables, se evaluó el territorio abarcado (grado de absorción) por la FAVEZ UPCH respecto a la captación de ingresantes, para ellos se emplearon las variables cualitativas lugar de nacimiento y residencia. Es evidente que la mayoría de ingresantes fueron de nacionalidad peruana. En relación al lugar de nacimiento de los ingresantes se obtuvo que el 92,4\% coincidió con la misma ciudad donde se encuentra la sede de la FAVEZ UPCH, Lima. Este resultado coincide con los datos nacionales, donde el $89,5 \%$ de alumnos de pre grado permanecen en el lugar de nacimiento para cursar estudios universitarios (INEI, 2011).

En principio se espera que el estudiante prefiera continuar estudios superiores en la universidad de mayor proximidad a su residencia. En la actualidad, la existencia de un gran número de universidades a nivel nacional ha permitido que la migración a la capital para seguir estudios superiores se vea disminuida. Prácticamente en todos los departamentos del Perú se encuentran universidades nacionales o particulares, que ofrecen una amplia variedad de carreras que permiten que los residentes de las ciudades no necesiten emigrar para estudiar (INEI, 2011). Para la 
UPCH, en el año 2010, el 73,5\% de alumnos de pre grado habían nacido en el mismo departamento de la universidad (INEI, 2011). Con respecto a la FAVEZ $\mathrm{UPCH}$, se mantuvo la misma tendencia durante el periodo de estudio, teniendo como lugar de residencia principalmente Lima Centro seguido del Cono Norte ambos próximos a la sede de la facultad.

Si bien la cercanía entre la universidad y el lugar de residencia llega a ser un factor importante en la elección de la casa de estudios, la presencia de ingresantes de otras regiones puede ser explicada por que ellos emplean otro criterio para seleccionar el lugar de estudio. Una encuesta realizada por el INEI (2011) indicó los otros criterios que emplean los postulantes, el prestigio de la universidad (54,0\%) era uno de los más importantes, seguidos en menor proporción por la especialidad que ofrece y la búsqueda de mejores expectativas económicas.

La mayor cantidad de ingresantes a la FAVEZ $\mathrm{UPCH}$, durante el periodo de estudio provienen de un colegio particular $(86,6 \%)$. Esta variable se puede relacionar con la condición socioeconómica de la familia, ya que los estudios de esta carrera en la UPCH tienen una pensión más costosa a diferencia de otras universidades que ofrecen la misma carrera. Esta proporción difiere del promedio nacional reportado por el INEI (2011) en donde se ha encontrado que los estudiantes de pregrado provienen en su mayoría de instituciones educativas nacionales con respecto a las privadas.

Como es de esperarse, en las universidades públicas la procedencia de estudiantes de instituciones educativas nacionales es mayor $(76,2 \%)$, sin embargo desde el punto de vista socioeconómico es menos concentrada que la educación superior privada (Díaz, 2008). No obstante, para las universidades privadas, los estudiantes procedentes de instituciones educativas nacionales también se encuentran en gran porcentaje $(54,0 \%)$. Sin embargo, esto no es el caso de la UPCH que durante el año 2010 solo recibió $26,3 \%$ de ingresantes provenientes de colegios nacionales (INEI, 2011). Este contraste con los resultados de los ingresantes a la FAVEZ UPCH se puede deber a que la presencia de muchas universidades particulares tienen pensiones de bajo costo, lo que permite ser accesible a un mayor público.

Además, se evaluó el porcentaje de beca por subvención económica asignada a los ingresantes de la facultad, ya que podría ser usada como una medida indirecta que permite una aproximación a las dimensiones socioeconómicas de la población en estudio. El mayor porcentaje lo obtuvo la población que no se ve beneficiada por ningún porcentaje de beca $(29,2 \%)$, seguida por la población que se encuentra en el rango de 41-50\% de beca. Según el INEI (2011), sólo el $13,7 \%$ se beneficia con algún tipo de beca en las universidades privadas $(96,4 \%$ de estudios, $3,1 \%$ alimentación, 2,0\% vivienda, 1,9\% otros), dato que no coincide con los resultados de este estudio, ya que para la FAVEZ-UPCH el $69,8 \%$ se ve beneficiado por algún porcentaje de beca en el periodo de estudio.

Esta situación puede ser importante ya que los aspectos culturales y socioeconómicos suelen estar asociados al rezago y el abandono de los estudiantes de educación superior. Ortega (2011) ha encontrado que los bajos ingresos familiares y la falta de estudios universitarios de los padres, aumentan la tasa de abandono. Así mismo, se encuentran asociados a una mayor tasa de repetición de cursos, a un menor avance en créditos por parte de los estudiantes y al incremento hasta de cuatro veces de la probabilidad de abandonar la universidad en el primer año de estudio, reduciendo casi a la mitad el porcentaje de obtención del grado.

Respecto a la modalidad de ingreso a la FAVEZ $\mathrm{UPCH}$ en el periodo de estudio, el mayor porcentaje correspondió a la modalidad factor excelencia, seguida de la modalidad examen de admisión. Estos resultados contrastan con los reportados a nivel nacional, el cual revela a la modalidad de ingreso por examen de admisión $(68,1 \%)$ como la más frecuente. Sin embargo, cuando se compara la modalidad de ingreso entre universidades nacionales y privadas, los primeros puestos tienen una incidencia algo mayor en acceder a las universidades privadas respecto a las públicas ( $6,4 \%$ y $5,1 \%$ respectivamente), mientras que el centro preuniversitario tiene mayor importancia en las universidades públicas que en las privadas $(22,7 \%$ y $10,1 \%$ respectivamente) (INEI, 2011).

Al evaluar la modalidad de ingreso respecto al tipo de colegio de procedencia, se mantuvo la misma tendencia, para ambos la modalidad factor excelencia fue de mayor elección por los ingresantes; sin embargo al ser comparadas con la modalidad examen de admisión la cual ocupó segundo lugar en preferencia, el grupo procedente de un colegio nacional sólo tuvo 1 individuo de diferencia entre las 2 modalidades, en contraste con el grupo procedente de un colegio particular que tuvo como diferencia a 85 individuos. 
No se encontró relación entre la profesión de medicina veterinaria de los padres con la elección de la carrera por parte de los ingresantes, ya que el porcentaje de padres MV no tuvo un valor significativo con respecto a otras profesiones.

En términos generales podemos concluir que la población ingresante para la FAVEZ UPCH va en aumento, al igual que el número de universidades a nivel nacional. Debido a este incremento de la competencia, la facultad se ve en la necesidad de determinar el potencial grupo poblacional específico que esté dispuesto a estudiar en la FAVEZ-UPCH, y así el trabajo de captación de ingresantes será focalizado. Este estudio logró identificar algunas características resaltantes de este grupo potencial.

Entre ellas encontramos que el género femenino es el porcentaje representativo de la muestra, la edad de ingreso es muy similar a la media nacional. La modalidad de ingreso más usada por los ingresantes fue factor excelencia, y el mayor porcentaje de la muestra se ve beneficiado por algún tipo de beca. Un dato revelador fue que los alumnos permanecen en el lugar de residencia para cursar sus estudios superiores, por lo que los esfuerzos de captación de estudiantes serían suficientes a nivel de la ciudad de Lima. Sin embargo, es necesario realizar estudios complementarios que identifiquen otras características que sean resaltantes de la población dispuesta a estudiar MVZ.

Se concluye que la población ingresante a la FAVEZ UPCH va en aumento. En cuatro años el número de esta población casi se ha duplicado. Los ingresantes a la FAVEZ durante el periodo de estudio fueron predominantemente de género femenino, la edad promedio de ingreso fue de 18,2 años, y gran parte reside en Lima. Los ingresantes de colegio particular representan el mayor porcentaje para la FAVEZ $\mathrm{UPCH}$, y la modalidad de ingreso más utilizada fue la de factor excelencia. El mayor porcentaje de los ingresantes FAVEZ UPCH se ve beneficiado por algún porcentaje de beca. La profesión de los padres no tiene una relación directa con la elección de la carrera de los hijos. Los ingresantes a la FAVEZ-UPCH en el periodo 2010-2013 no presentan un perfil homogéneo y definido.

\section{Correspondencia:}

Ana Lucia Paredes Ramos ana.paredes@upch.pe

\section{REFERENCIAS BIBLIOGRÁFICAS}

1. [APOYO S.A.] APOYO Opinión y Mercado S.A. 2006. El informe Perfil del Mercado Educativo Postulantes 2006. Grupo APOYO S.A. 125p.

2. Bertoni E. 2005. El estudiante universitario - Una aproximación al perfil de ingreso. Comisión Sectorial de Enseñanza. $37 \mathrm{p}$.

3. Díaz J. 2008. Educación Superior en el Perú: Tendencias de la demanda y oferta. Grupo de Análisis para el Desarrollo [GRADE]. 83 - 129p.

4. Grupo Educación al Futuro e Investy S.A.C. 2013. Guía Vocacional. 12ava edición. Estudio Digital Editores S.A.C. 229p.

4. Instituto Nacional de Estadística e Informática. 2011. II Censo Universitário Nacional 2010. Instituto Nacional de Estadística e Informática INEI. 456p.

5. Instituto Nacional de Estadística e Informática. 2013. Perú: Indicadores de Educación por Departamentos, 2001 - 2011. Capitulo 5: Profesiones o carreras universitarias. Instituto Nacional de Estadística e Informática- INEI. 107$118 \mathrm{p}$.

6. Mikkonen J., Heikkila A., Ruohoniemi M., Lindblom-Ylanne S., 2009. I Study Because I'm Interested. University Students Explanations for Their Disciplinary Choices. Scandinavian Journal of Educational Research 53 (3): 229-244p.

7. Ministerio de Educación. 2006. La Universidad en el Perú - Razones para la Reforma Universitaria Informe 2006. Dirección de Coordinación Universitaria-Ministerio de Educación. 198p.

8. Ortega M. 2011. Aspectos Vocacionales del perfil de los estudiantes de primer ingreso a la Facultad de Medicina Veterinaria y Zootecnia (FMVZ) de la UNAM: Generación 2011. XI Congreso Nacional de Investigación Educativa - Sujetos de la Educación. 14p.

9. Pallarés D. 2011. La neurociencia aplicada al estudio de género: ¿una nueva perspectiva? Fórum de Recerca. 16p.

10. Taylor J. 2012. Competencias profesionales en Medicina Veterinaria. PANVET. 162p. 